November 13, 2018

\title{
The diffractive structure function at the Tevatron: CDF results
}

\author{
K. Goulianos ${ }^{\mathrm{a} *}$ \\ aThe Rockefeller University, \\ 1230 York Avenue, New York, NY 10021, USA \\ (dino@physics.rockefeller.edu)
}

Results on hard diffraction from CDF are reviewed with emphasis on the determination of the diffractive structure function of the (anti)proton from single diffractive and double Pomeron exchange dijet production. Comparison of the diffractive dijet results with predictions based on diffractive deep inelastic scattering at HERA shows a breakdown of conventional QCD factorization. A similar breakdown is observed within the CDF results in comparing the structure functions obtained from single diffraction and double Pomeron exchange.

\section{INTRODUCTION}

Hadronic diffraction [1] is believed to be mediated by the exchange of the Pomeron [2]. In the framework of QCD, the Pomeron consists of (anti)quarks and gluons in a color-singlet state with vacuum quantum numbers. An interesting question is whether the Pomeron, although virtual, has a unique partonic structure obeying QCD factorization, as is the case for real hadrons. Such a structure could be probed in diffractive processes which incorporate a hard scattering. Figure 1 shows the event topology for dijet production in single diffraction (SD), double diffraction (DD), and double Pomeron exchange (DPE). (a)

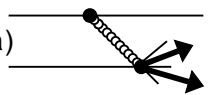

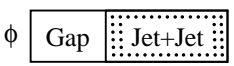

(b)
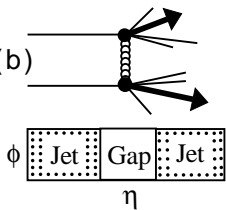

(c)

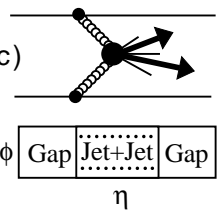

Figure 1. Dijet production diagrams and event topologies for (a) single diffraction (b) double diffraction and (c) double Pomeron exchange.

Since there is no color exchanged between the colorless Pomeron and the parent nucleon, a rapidity gap (region devoid of particles) emerges as a characteristic signature of Pomeron exchange, as shown schematically in the $\eta-\phi$ plots of Fig. 1.

\footnotetext{
* Representing the CDF Collaboration at "Diffraction 2000, Cetraro, Italy, 2-7 September 2000".
}

Such gaps can be used to tag diffractive production. Another way of tagging diffraction is provided by the recoil $\bar{p}$ or $p$ in $\mathrm{SD}$ or in DPE. The CDF Collaboration has reported results for hard diffraction in $\bar{p} p$ collisions at $\sqrt{s}=1800 \mathrm{GeV}$ obtained by using both tagging techniques 3 .7.

At HERA, where $\sim 28 \mathrm{GeV}$ electrons are brought into collision with $\sim 800 \mathrm{GeV}$ protons $(\sqrt{s} \approx 300$ $\mathrm{GeV}$ ), diffraction has been studied both in photoproduction and in high $Q^{2}$ deep inelastic scattering (DIS). The $\mathrm{H} 1$ and ZEUS Collaborations have measured the diffractive structure function of the proton and its factorization properties [8,9]. Figure 2 shows the kinematics of a DIS diffractive collision.

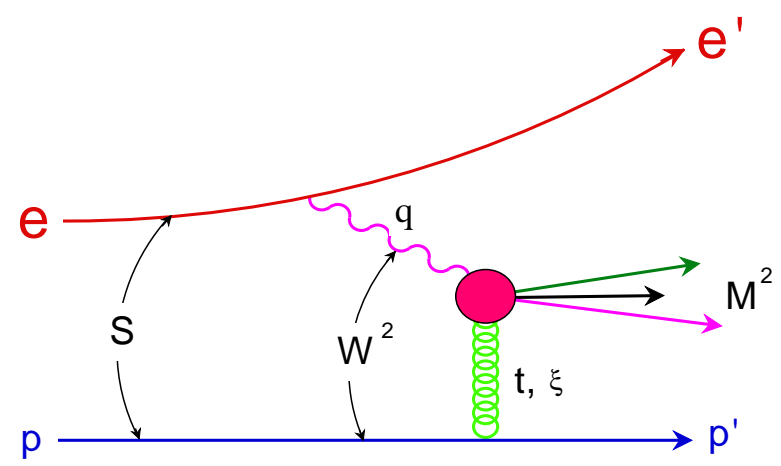

Figure 2. Schematic diagram of a diffractive DIS collision involving a virtual photon, emitted by an electron, and a virtual Pomeron, emitted by a proton. 
In analogy with the $F_{2}\left(Q^{2}, x\right)$ structure function, the 4-variable diffractive structure function (DSF) of the proton, $F_{2}^{D(4)}\left(Q^{2}, x, \xi, t\right)$, is defined through the cross section equation

$$
\frac{d^{4} \sigma}{d Q^{2} d x d \xi d t}=\frac{4 \pi \alpha^{2}}{x Q^{4}} \cdot f(y) \cdot F_{2}^{D(4)}\left(Q^{2}, x, \xi, t\right)(1)
$$

where $x$ and $y$ are the Bjorken DIS variables, $f(y) \equiv 2-2 y+y^{2} /[2(1+R)]$, and $\xi$ is the fraction of the proton's momentum carried by the Pomeron. The $t$-integrated DSF, $F_{2}^{D(3)}$, has been determined from the data by assuming $R=0$ in $f(y)$. The reported measured values for $F_{2}^{D(3)}$ correspond to cross sections given by

$\frac{d^{3} \sigma}{d Q^{2} d \beta d \xi} \stackrel{|t|<1}{\equiv} \frac{4 \pi \alpha^{2}}{\beta Q^{4}} \cdot f(y) \cdot F_{2}^{D(3)}\left(Q^{2}, \beta, \xi\right)$

where $\beta \equiv x / \xi$ represents the fraction of the momentum of the Pomeron carried by the interacting quark.

Diffractive quark densities are derived directly from $F_{2}^{D(3)}$. Using a QCD analysis, the H1 Collaboration has also derived [8] diffractive gluon densities from the $Q^{2}$ dependence (scaling violations) of $F_{2}^{D(3)}$. In this paper, we present CDF results on single-diffractive to non-diffractive (ND) ratios for $W$, dijet, $b$-quark and $J / \psi$ production measured by using rapidity gap tagging, as well for dijet production using leading $\bar{p}$ tagging, and compare them with expectations based on diffractive parton densities extracted from HERA measurements to test factorization. In addition, factorization is tested within the CDF data by comparing dijet production in SD and in DPE.

\section{RESULTS USING RAPIDITY GAPS}

Using the rapidity gap method to tag diffraction, four hard SD processes have been studied by CDF: Diffractive $W$ [3], dijet [4], $b$-quark [5] and $J / \psi$ production.

The components of the CDF detector relevant to forward rapidity gap tagging are [10] the BeamBeam Counters (BBC) and the forward calorimeters (FCAL). The BBC consist of square arrays of 16 scintillation counters placed at $\pm z$ positions of $6 \mathrm{~m}$ from the center of the detector. The FCAL have a tower geometry with segmentation of 0.1 units in $\eta$ and $5^{\circ}$ in $\phi$. These detectors cover the $\eta$-range:

BBC $\quad 3.2<|\eta|<5.9$

FCAL $\quad 2.4<|\eta|<4.2$

Experimentally, a "particle" is defined as a hit in the BBC or a calorimeter tower with energy $E>1.5 \mathrm{GeV}$. The tower energy threshold is imposed to reduce calorimeter noise and to enhance the correspondence between a single tower above threshold and a real particle. In a sample of $W$, dijet, $b$-quark or $J / \psi$ events, the SD signal is identified as an excess of events above a ND "background" in the $(0,0)$ bin of the BBC versus FCAL multiplicity distribution. The ratio of SD to ND events is then evaluated, compared with Monte Carlo simulations, and conclusions are drawn about the Pomeron structure function and its factorization properties. Below, we briefly present the results obtained for each process studied by CDF and the conclusions drawn about the Pomeron structure.

\subsection{Diffractive $W$ production}

CDF made the first observation [3] of diffractive $W \mathrm{~s}$ and measured the $W$ production rate using a sample of 8246 events with an isolated central $e^{+}$or $e^{-}(|\eta|<1.1)$ of $E_{T}>20 \mathrm{GeV}$ and missing transverse energy $\mathbb{E}_{T}>20 \mathrm{GeV}$. In searching for diffractive events, CDF studied the correlations of the BBC multiplicity, $N_{B B C}$, with the sign of the electron- $\eta, \eta_{e}$, or the sign of its charge, $C_{e}$. In a diffractive $W^{ \pm} \rightarrow e^{ \pm} \nu$ event produced in a $\bar{p}$ collision with a Pomeron emitted by the proton, a rapidity gap is expected at positive $\eta$ ( $p$-direction), while the lepton is boosted towards negative $\eta$ (angle-gap correlation). Also, since the Pomeron is quark-flavor symmetric, and since, from energy considerations, mainly valence quarks from the $\bar{p}$ participate in producing the $W$, approximately twice as many electrons as positrons are expected (charge-gap correlation).

Figure 3 shows the BBC versus tower multiplicity for two event samples characterized by the correlation between the pseudorapidity of the BBC whose multiplicity is plotted, $\eta_{B B C}$, and the $\eta_{e}$ or $C_{e}$, as follows: (a) doubly-correlated events, for which $\eta_{e} \cdot C_{e}>0$ and $\eta_{e} \cdot \eta_{B B C}<0$, 
and (b) doubly-anticorrelated events, for which $\eta_{e} \cdot C_{e}>0$ and $\eta_{e} \cdot \eta_{B B C}>0$. Monte Carlo simulations show that diffractive $W$ events are expected to have low BBC or tower multiplicities (in the range 0-3), and that there should be about 4 times as many doubly-correlated than doublyanticorrelated events. This diffractive signature is satisfied by the small number of events at low multiplicities in Fig. 3 .
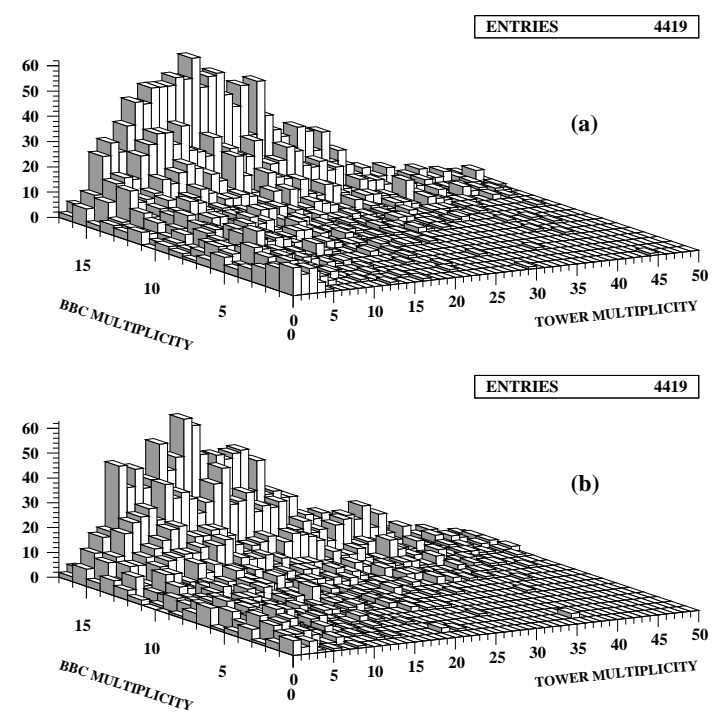

Figure 3. Tower versus BBC multiplicity for $W$ events: (a) (angle $\otimes$ charge)-correlated; (b) (angle $\otimes$ charge)-anticorrelated.

Correcting for acceptance, the ratio of diffractive to non-diffractive $W$ production is:

$R_{W}=[1.15 \pm 0.51($ stat $) \pm 0.20($ syst $)] \%(\xi<0.1)$ Figure 4 shows Pomeron- $\xi$ distributions of $W$ events generated by the POMPYT [11] Monte Carlo program followed by a detector simulation.

\subsection{Diffractive dijet production}

CDF searched for diffractive dijet production in a sample of 30352 dijet events with a single-vertex (to exclude events from multiple interactions), in which the two leading jets have $E_{T}>20 \mathrm{GeV}$ and are both at $\eta<1.8$ or $\eta>1.8$. No requirement was imposed on the presence or kinematics of extra jets in an event. Figure a shows the

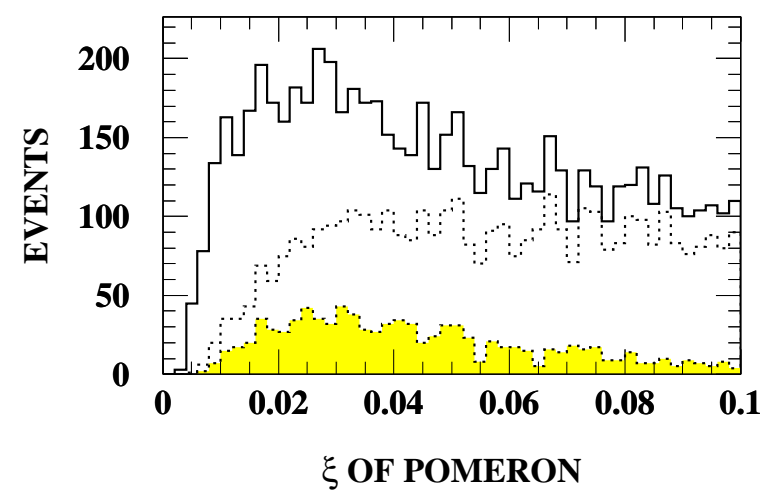

Figure 4. Monte Carlo Pomeron $\xi$ distributions for diffractive $W$ events generated by POMPYT using a hard-quark Pomeron structure: (solid line) all events; (dotted line) events with a central electron; (shaded area) events with a central electron and 0,1 or 2 hits in the (angle $\oplus$ charge)correlated BBC (corresponding to the signal).

correlation of the BBC and forward $(|\eta|>2.4)$ calorimeter tower multiplicities in the $\eta$-region opposite the dijet system. The excess in the $0-0$ bin is attributed to diffractive production. After subtracting the non-diffractive background and correcting for the single-vertex selection cut, for detector live-time acceptance and for the rapidity gap acceptance $(0.70 \pm 0.03)$, calculated using the POMPYT Monte Carlo program with Pomeron $\xi<0.1$, the "Gap-Jet-Jet" fraction (ratio of diffractive to non-diffractive dijet events) was found to be

$$
\begin{gathered}
R_{G J J}=[0.75 \pm 0.05(\text { stat }) \pm 0.09(\text { syst })] \%= \\
(0.75 \pm 0.10) \% \\
\left(E_{T}^{j e t}>20 \mathrm{GeV},|\eta|^{j e t}>1.8, \eta_{1} \eta_{2}>0, \xi<0.1\right)
\end{gathered}
$$

Figure 6 shows Pomeron- $\xi$ distributions of dijet events generated by a POMPYT Monte Carlo simulation for $\xi<0.1$ using a hard gluon Pomeron structure. The jets were required to have $E_{T}^{\text {jet }}>20 \mathrm{GeV}$ and be in the region $1.8<$ $|\eta|<3.5$ with $\eta_{1} \cdot \eta_{2}>0$.

\subsection{Diffractive $b$-quark production}

Diffractive $b$-quark production probes directly the gluon content of the Pomeron. In a sample of events collected in the 1994-95 run (80 


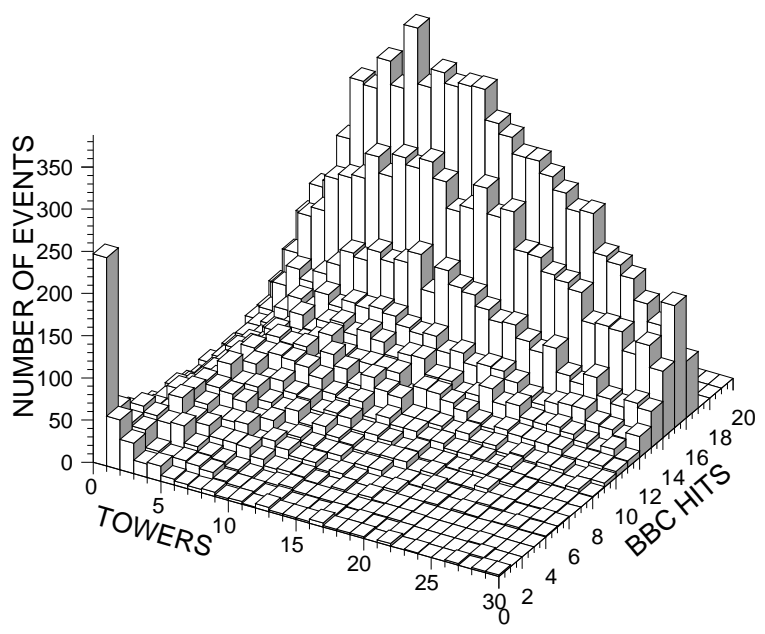

Figure 5. FCAL tower versus BBC multiplicity on the opposite hemisphere of the dijet system for dijet events with both jets at $\eta>1.8$ or $\eta<1.8$.

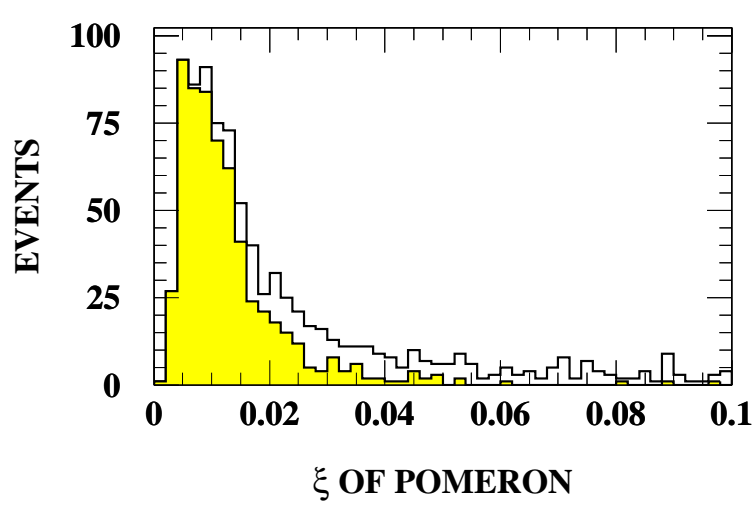

Figure 6. Monte Carlo Pomeron $\xi$ distributions for diffractive dijet events with jet $E_{T}>20 \mathrm{GeV}$ and $1.8<|\eta|<3.5$ generated by POMPYT using a hard-gluon Pomeron structure. The shaded area represents the subset of Monte Carlo events with zero BBC and forward calorimeter multiplicities, corresponding to the data in the $(0,0)$ bin of Fig. .
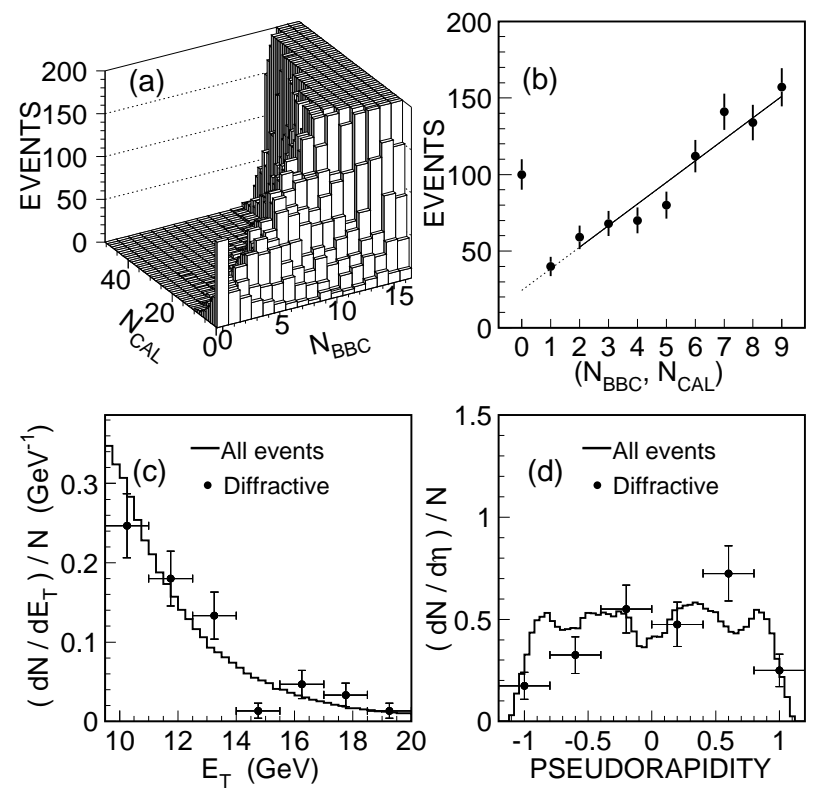

Figure 7. Diffractive $b$-quark production: (a) Forward calorimeter tower multiplicity, $N_{C A L}$, versus beam-beam counter multiplicity, $N_{B B C}$; (b) multiplicity distribution along the diagonal with $N_{B B C}=N_{C A L}$ in the plot in (a); (c) electron $E_{T}$; and (d) pseudorapidity for the diffractive (points) and total (histogram) event samples (diffractive events with a rapidity gap at positive $\eta$ are entered with the sign of the electron $\eta$ changed).

$\mathrm{pb}^{-1}$ ) with a trigger requiring an electron of $E_{T}^{e}>7.5 \mathrm{GeV}$ within $|\eta|<1.1$, CDF searched for diffractive events after imposing the software cuts of $E_{T}^{e}>9.5 \mathrm{GeV}$ (to avoid trigger bias) and $E_{T}^{e}<20 \mathrm{GeV}$ (to reject $W$ and $Z$ boson events. As in the previous studies, the diffractive signal was evaluated from the FCAL versus $\mathrm{BBC}$ multiplicity distribution, which is shown in Fig. 7a. The excess of events in the $(0,0)$ bin above a smooth extrapolation from nearby bins is attributed to diffractive events. The ND content of the $(0,0)$ bin was evaluated from the distribution of events along the diagonal of Fig. Fa with $N_{B B C}=N_{C A L}$, which is shown in Fig. 值. Figures $7 \mathrm{c}$ and $7 \mathrm{~d}$ show the electron $E_{T}$ and $\eta$ distributions, respectively, for the diffractive and total event samples.

After extracting the $b$-quark content from 


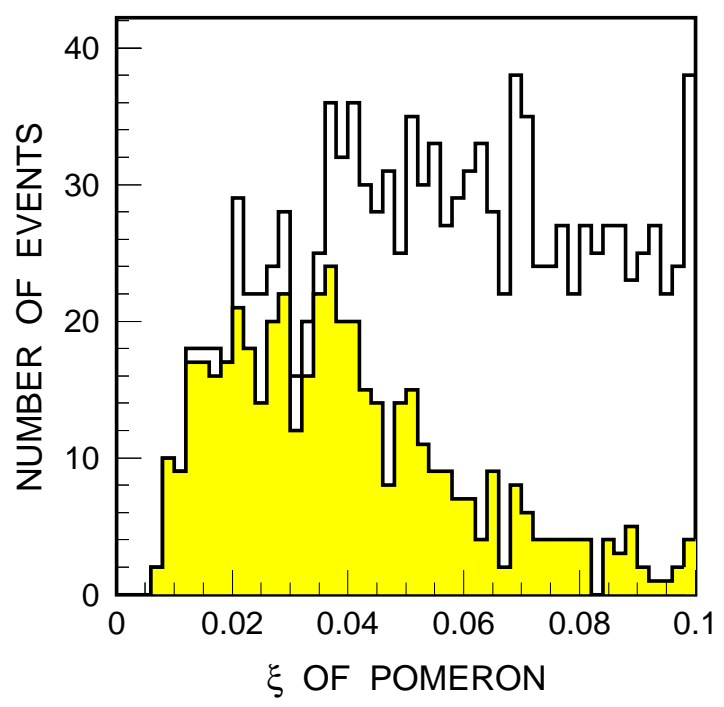

Figure 8. Monte Carlo distribution of the Pomeron beam momentum fraction, $\xi$, for diffractive $b$-quark production events with an electron of $9.5<p_{T}^{e}<20 \mathrm{GeV} / c$ within $|\eta|<1.1$, generated using a flat Pomeron structure of gluon to quark ratio $0.7 \div 0.3$. The shaded area is the distributions for events satisfying the rapidity gap requirements.

the event sample, separately for the SD and ND events, and correcting for various backgrounds [5], the ratio of SD to ND $b$ production was found to be

$$
R_{\bar{b} b}^{\text {gap }}=[0.23 \pm 0.07(\text { stat }) \pm 0.05(\text { syst })] \%
$$

The rapidity gap acceptance for events generated with a flat Pomeron structure, which is approximately the structure obtained from the HERA measurements [8,9], and a gluon fraction of $0.7 \pm 0.2$, as reported in [4], is found to be $0.37 \pm 0.02$. Dividing $R_{\bar{b} b}^{\text {gap }}$ by this value yields a diffractive to total production ratio of

$$
\begin{gathered}
R_{\bar{b} b}=[0.62 \pm 0.19(\text { stat }) \pm 0.16(\text { syst })] \% \\
\left(9.5<E_{T}^{E}<20 \mathrm{GeV} \quad\left|\eta^{e}\right|<1.1 \quad \xi<0.1\right)
\end{gathered}
$$

Fig. 8 shows Pomeron- $\xi$ distributions of $b$ events generated by the POMPYT Monte Carlo program followed by a detector simulation.

\subsection{Diffractive $J / \psi$ production}

As in the case of diffractive $b$-quark production, diffractive $J / \psi$ production probes directly the gluon content of the Pomeron (see Fig. 9). The $J / \psi$ study is interesting because, since muons from $J / \psi \rightarrow \mu^{+} \mu^{-}$can be detected in CDF down to $p_{T} \sim 2 \mathrm{GeV}$, the $\mathrm{SD}$ to ND fraction can be measured at $p_{T}$ values about five times lower than those in the $b$-quark case. Thus, from the combined $J / \psi$ and $b$-quark measured fractions one may determine the $p_{T}$ dependence of the gluon fraction of the Pomeron. Moreover, the leading (anti)proton momentum fraction of the parton participating in $J / \psi$ production, $x_{b j}$, can be determined from the measured vector momenta of the $J / \psi$ and the accompanying jet. This allows one to determine the fraction of SD to ND production as a function of $x_{b j}$. Figure 10 shows the

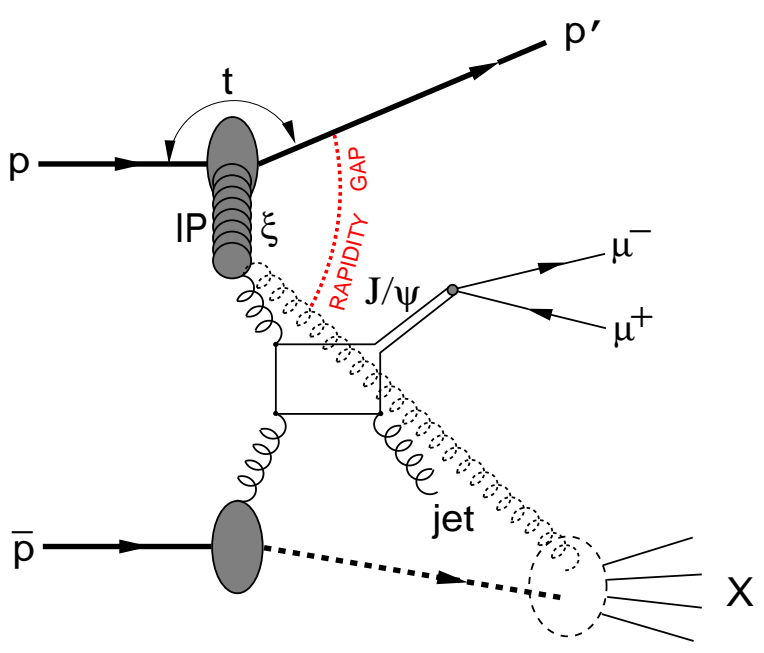

Figure 9. Schematic diagram of diffractive $J / \psi$ production. Note the jet in the final state, which is expected to balance the $J / \psi$ momentum in diffractive events.

same distributions as those in Fig. 1 but for $J / \psi$ rather than $b$-quark events. The $J / \psi$ is identified by its decay into two muons, $J / \psi \rightarrow \mu^{+} \mu^{-}$. The events selected have muons of $p_{T}^{\mu}>2 \mathrm{GeV}$ within $|\eta|<1$.1. The SD to ND fraction for events with a forward rapidity gap is found to be

$$
R_{J / \psi}^{\text {gap }}=[0.36 \pm 0.06(\text { stat })] \%
$$


6
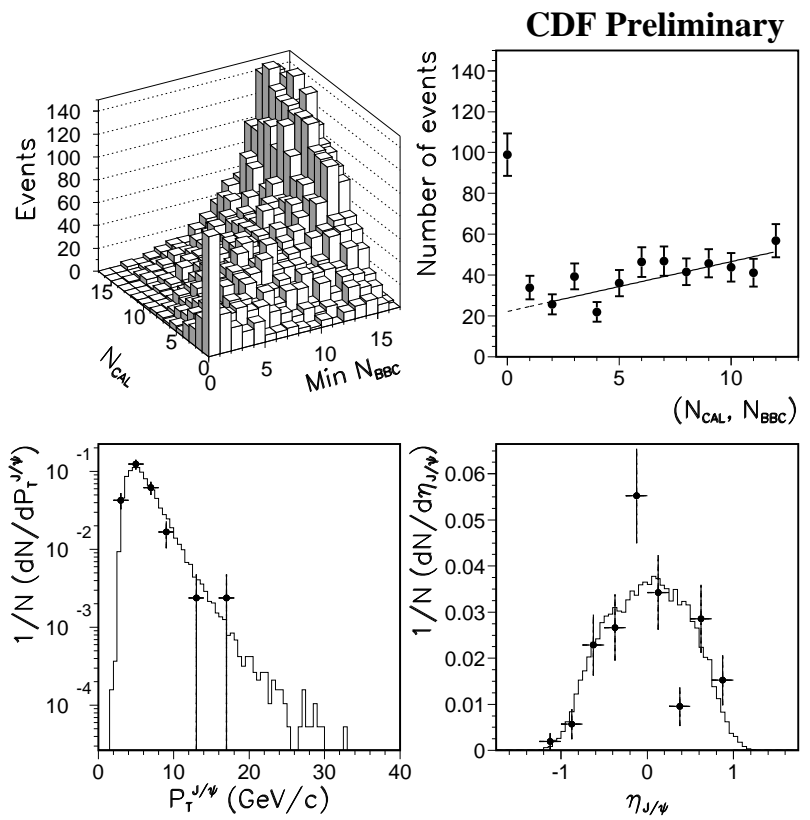

Figure 10. Diffractive $J / \psi$ production: (a) Forward calorimeter tower multiplicity, $N_{C A L}$, versus beam-beam counter multiplicity for the $\eta$ side of the event with the minimum BBC multiplicity, Min $N_{B B C}$; (b) multiplicity distribution along the diagonal with $N_{B B C}=N_{C A L}$; (c) $p_{T}$ of $J / \psi$, and (d) pseudorapidity for the diffractive (points) and total (histogram) event samples (diffractive events with a rapidity gap at positive $\eta$ are entered with the sign of the electron $\eta$ changed).

This value is is about 1.5 times larger than the measured SD to ND $b$-quark fraction, $R_{\bar{b} b}^{\text {gap }}$. Under the reasonable assumption that the gap acceptances are similar for the two cases, the larger $R$ value measured in $J / \psi$ production must be attributed to the lower $p_{T}$ of the hard scattering.

\subsection{Gluon fraction and factorization}

By combining the diffractive $W$, dijet and $b$ quark results, $\mathrm{CDF}$ extracted the gluon fraction of the Pomeron, $f_{g}$. Assuming the standard Pomeron flux in the POMPYT Monte Carlo program, the measured $W$, dijet and $b$-quark SD to ND ratios trace curves in the plane of $D$ versus $f_{g}$, where $D$ is the ratio of measured to POMPYT-predicted rates. Figure 11 shows the $\pm 1 \sigma$ curves corresponding to the results. From the oval-shaped overlap of the $W$, dijet and $b$ quark curves, CDF obtained $f_{g}=0.54_{-0.14}^{+0.16}$. This

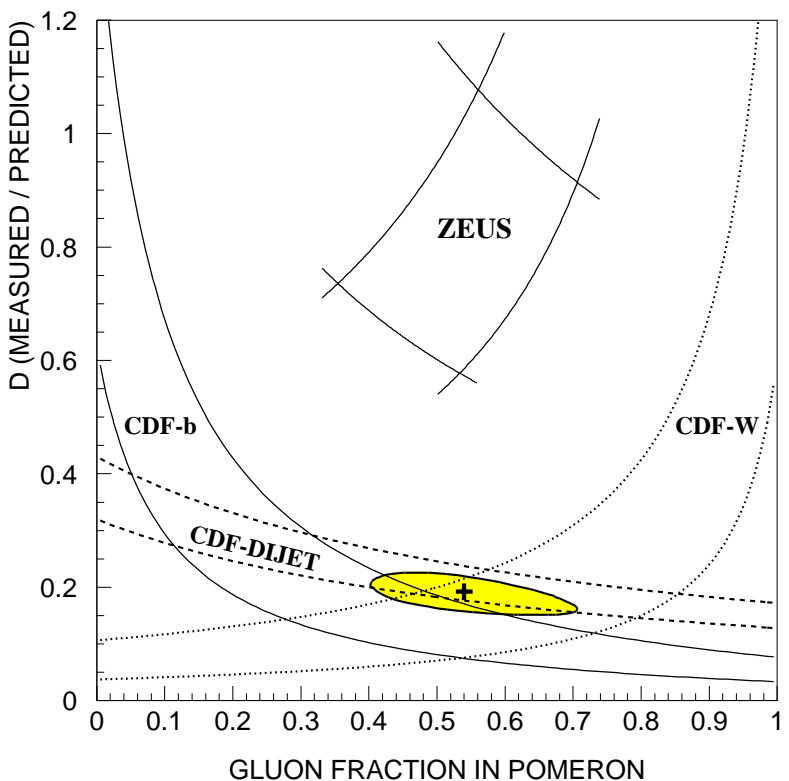

Figure 11. The ratio, $D$, of measured to predicted diffractive rates as a function of the gluon content of the Pomeron. The predictions are from POMPYT using the standard Pomeron flux and a hard Pomeron structure. The CDF- $W$ curves were calculated assuming a three-flavor quark structure for the Pomeron. The black cross and shaded ellipse are the best fit and $1 \sigma$ contour of a least square two-parameter fit to the three CDF results.

result, which is independent of the Pomeron flux normalization, agrees with the result obtained by ZEUS [9] from DIS and dijet photoproduction (diamond-shaped area shown in the figure). For the $D$-fraction, CDF obtained the value $D=$ $0.19 \pm 0.04$. The decrease of the $D$-fraction from HERA to the Tevatron represents a breakdown of factorization.

The observed factorization breakdown can be further characterized by measuring the shape of the diffractive structure function at the Tevatron and comparing it with predictions based on the HERA measurements. This has been done by CDF in studies of dijet production in SD and DPE using leading particle tagging, as described in the next section. 


\section{RESULTS USING ROMAN POTS}

Using a Roman Pot Spectrometer (RPS) to trigger on and measure the momentum of leading antiprotons, CDF collected about 3,000 K events at $\sqrt{s}=1800 \mathrm{GeV}$ with no other requirement on the trigger. After requiring a single vertex within $\left|z_{v t x}\right|<60 \mathrm{~cm}$, to reject events due to more than one interactions, and applying quality cuts on the RPS tracks, there remained $1,639 \mathrm{~K}$ events in the region $0.035<\xi_{\bar{p}}<0.095$ and $|t|<1 \mathrm{GeV}^{2}$. This inclusive diffractive event sample contains 30,639 events with two jets of $E_{T}^{\text {jet }}>7 \mathrm{GeV}$. A non-diffractive event sample was also collected by requiring only a BBC coincidence. This Minimum Bias (MB) sample consists of 299,959 events with $\left|z_{v t x}\right|<60 \mathrm{~cm}$ and contains 32,629 dijet events of $E_{T}^{\text {jet }}>7 \mathrm{GeV}$. The above event samples were used for measuring the diffractive structure function of the antiproton in SD events, as well as that of the proton in DPE. Diffractive factorization was tested by comparing these structure function to each other and with predictions based on HERA measurements, as described below.

\subsection{Single-diffractive dijets}

The diffractive dijet sample was used to measure the diffractive structure function (DSF) of the antiproton [6]. The procedure used, designed to avoid Monte Carlo simulations, is based on measuring the ratio $R(x)$ of SD to ND cross sections as a function of the Bjorken $x$ of the parton in the $\bar{p}$ participating in the hard scattering. In LO QCD, this ratio is proportional to the corresponding structure functions. The DSF is then obtained by multiplying the measured $R(x)$ by the known ND structure function. The absolute normalization of the SD dijet sample is obtained by scaling the event rate to that of the inclusive diffractive sample and using for the latter the previously measured inclusive cross section [12]. The normalization of the ND dijet sample is obtained from the measured $51.2 \pm 1.7 \mathrm{mb}$ cross section of the $\mathrm{BBC}$ trigger.

The variable $x$ is evaluated from the jet $E_{T}$ and $\eta$, as follows:

$$
x=\frac{1}{\sqrt{s}} \sum_{i=1}^{n} E_{T}^{i} e^{-\eta^{i}}
$$

The sum is carried over the two leading jets plus the next highest $E_{T}$ jet, if there is one with $E_{T}>$ $5 \mathrm{GeV}$.
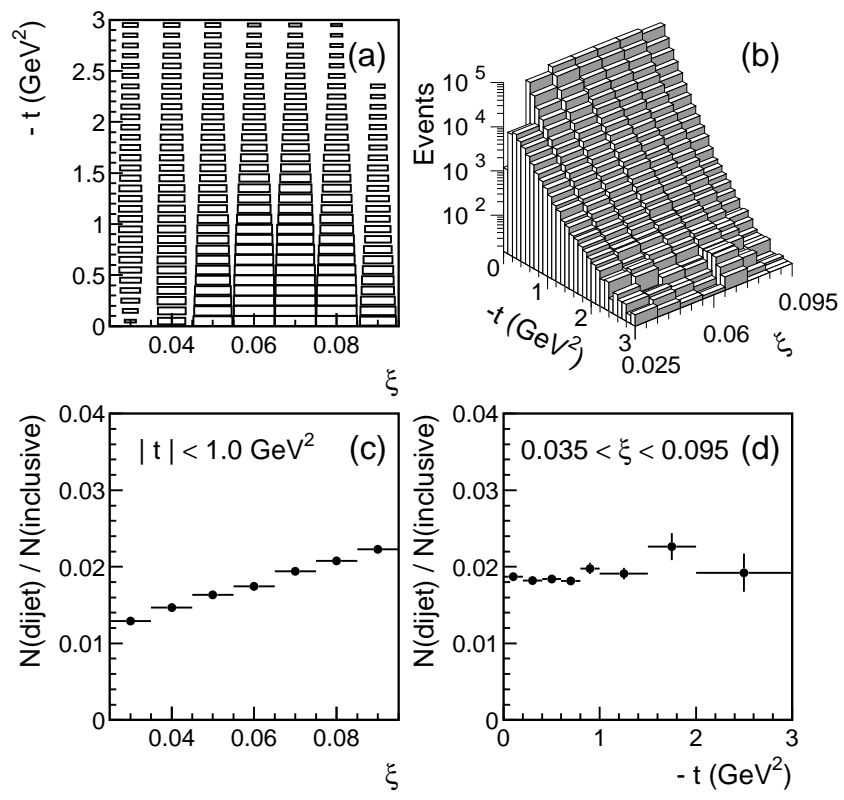

Figure 12. Distributions versus $\xi$ and $t$ : (a) Roman pot acceptance; (b) inclusive diffractive event sample; (c) ratio of dijet to inclusive diffractive events versus $\xi$ and (d) versus $t$.

The structure function relevant to dijet production is a color-weighted combination of quark and gluon components

$$
F_{j j}(x)=x\left\{g(x)+\frac{4}{9} \sum_{i}\left[\left(q_{i}(x)+\bar{q}_{i}(x)\right]\right\}\right.
$$

where $g(x)$ and $q(x)$ are gluon and quark parton densities, respectively. For comparisons with predictions based on HERA results, in which the DSF is usually presented in terms of the variable $\beta$ instead of $x$, the DSF obtained from the equation $F_{j j}^{D}(x, \xi)=R(x, \xi) \times F_{j j}^{N D}(x)$ may be transformed to $F_{j j}^{D}(\beta, \xi)$ by a change of variables $(x=\beta \xi)$.

Results of the diffractive dijet analysis are presented in Figs. 12, 13 and 14 . The highlights are: (i) In Fig. 12d, the ratio of SD dijet to inclusive production rates is seen to be independent of $t$; (ii) In Fig. 13, the ratio of SD to ND dijet production rates exhibits a power law dependence for 


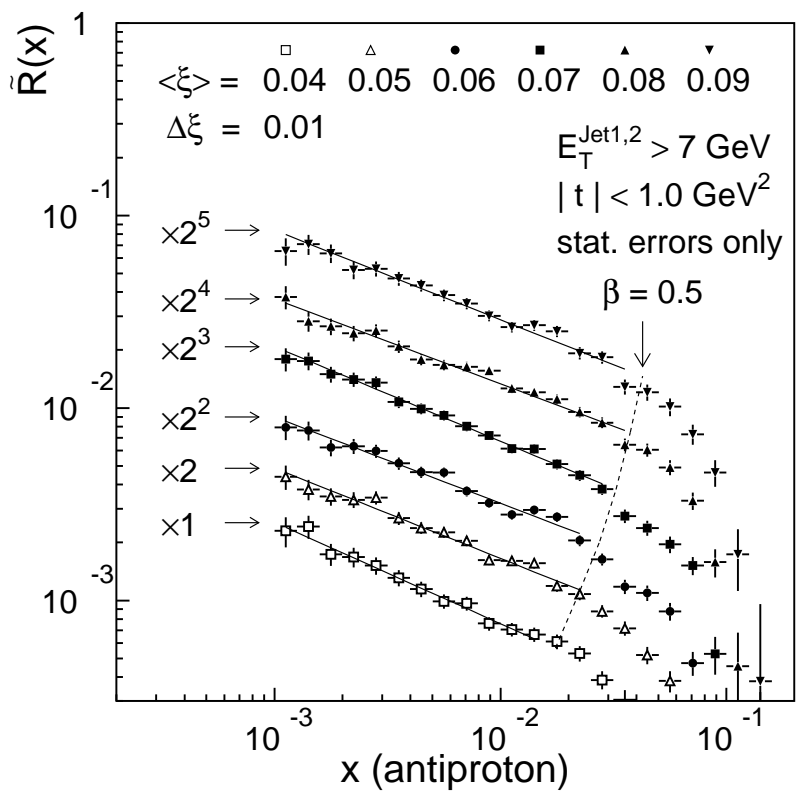

Figure 13. Ratio of diffractive to non-diffractive dijet event rates as a function of $x$ (momentum fraction of parton in $\bar{p})$. The solid lines are fits to the form $\tilde{R}(x)=R_{\circ}(x / 0.0065)^{-r}$ for $\beta<0.5$.

$\beta<0.5$, increasing with decreasing $x$ as $x^{-0.46}$; (iii) In Fig. 14, the diffractive structure function measured by CDF differs both in shape and normalization from expectations based on diffractive parton densities extracted by the H1 Collaboration from diffractive DIS measurements. The normalization discrepancy, which is of $\mathcal{O}(0.1)$, confirms the breakdown of factorization observed in the comparison of the rapidity gap results with expectations from HERA measurements.

\subsection{Double-Pomeron dijets}

Using the RPS single-diffractive dijet event sample, CDF searched for and discovered [7] events with a rapidity gap on the proton side, presumed to be due to the double Pomeron exchange process illustrated in Fig. 15b.

The DPE signal appears as an enhancement in the $(0,0)$ bin of the $N_{F C A L p}$ versus $N_{B B C p}$ distribution of Fig. 16a, and in the one-dimensional "diagonal" distribution of Fig. 16b. The $\xi_{p}$ distribution for the events of the $(0,0)$ bin of Fig. 16a is shown in Fig. 16 d. The $\xi_{p}$ values were determined from the calorimeter and BBC information [7].

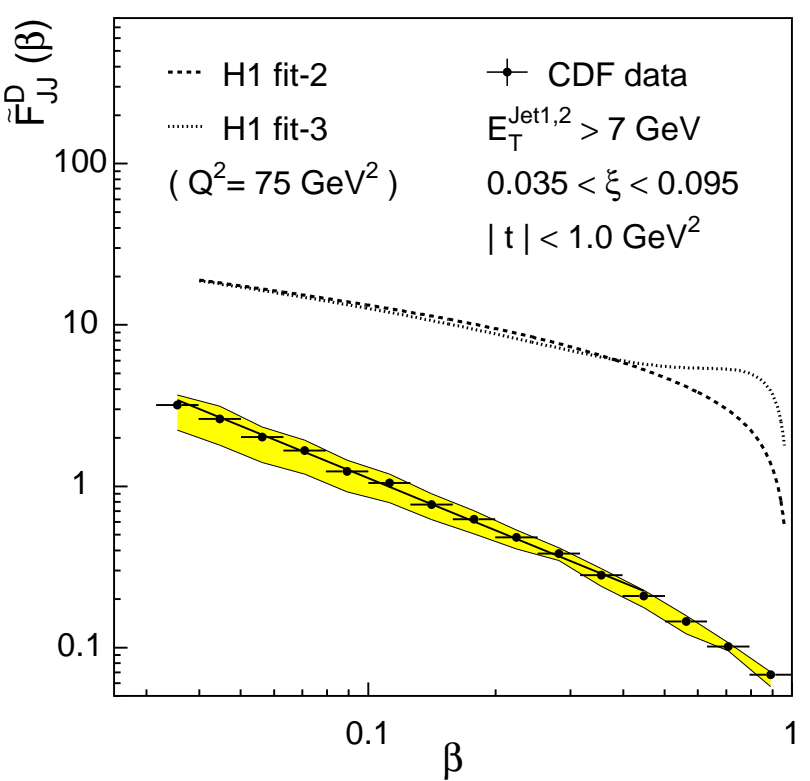

Figure 14. Data $\beta$ distribution (points) compared with expectations from the parton densities of the proton extracted from diffractive deep inelastic scattering by the H1 Collaboration at HERA.

In events with a leading antiproton, or equivalently with a rapidity gap on the $\bar{p}$ side, the ratio of the DPE to SD dijet production cross sections at the same $x_{p}$ for fixed $\xi_{p}, R_{S D}^{D P E}\left(x_{p}, \xi_{p}\right)$, is in LO QCD equal to the ratio of the SD to ND structure functions of the proton. Therefore, diffractive factorization can be tested by comparing this ratio with the SD to ND ratio, $R_{N D}^{S D}\left(x_{p}, \xi_{p}\right)$, for $\mathrm{SD}$ events with no rapidity gap on the antiproton side. Since no such events are available, the comparison was made with the measured [6] ratio $R_{N D}^{S D}\left(x_{p}, \xi_{\bar{p}}\right)$. The result is shown in Fig. 17. The vertical dashed lines mark the DPE kinematic boundary (left) and the value of $x=\xi_{p}^{\text {min }}$ (right). The weighted average of the DPE/SD points in the region within the vertical dashed lines is $\tilde{R}_{S D}^{D P E}=0.80 \pm 0.26$. As mentioned above, factorization demands that $\tilde{R}_{S D}^{D P E}$ be the same as $\tilde{R}_{N D}^{S D}$ at fixed $x$ and $\xi$. Since the $\xi_{p}$ and $\xi_{\bar{p}}$ regions, which are respectively relevant for the DPE/SD and $\mathrm{SD} / \mathrm{ND}$ ratios, do not overlap, we examine in the inset of Fig. 17 the $\xi$ dependence of the ratios $\tilde{R}(x)$ (per unit $\xi$ ), where the tilde over the $R$ indicates the weighted average of the points in the 

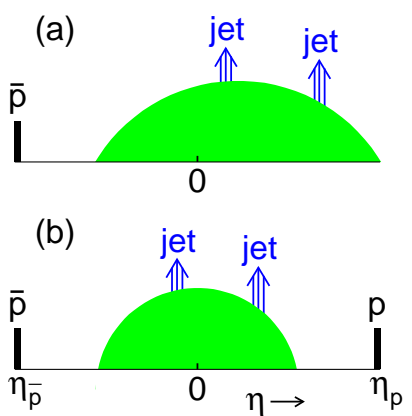

Figure 15. Illustration of event topologies in pseudorapidity, $\eta$, and associated Pomeron exchange diagrams for dijet production in (a) single diffraction and (b) double Pomeron exchange. The shaded areas on the left side represent particles not associated with the jets (underlying event).
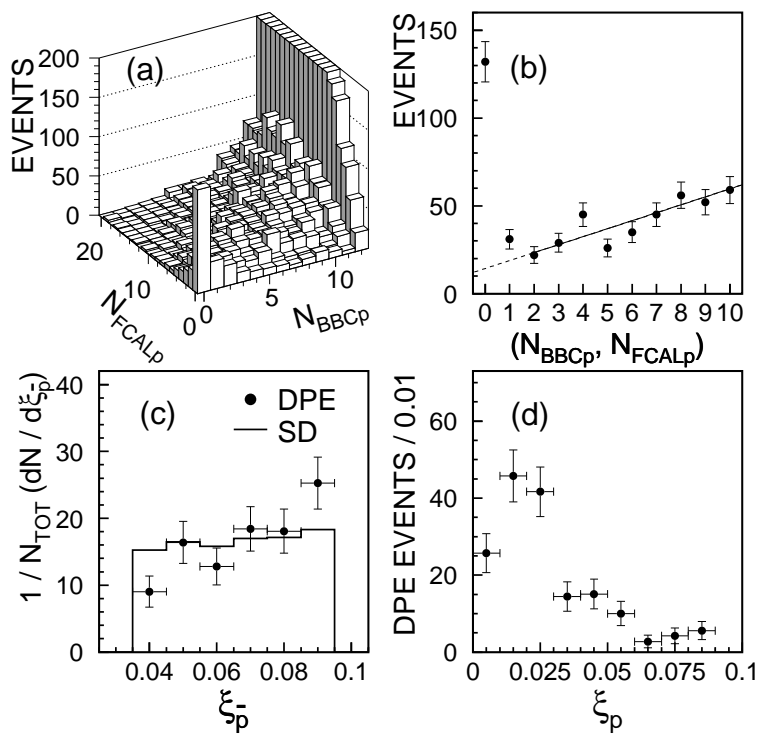

Figure 16. (a) Beam-Beam Counter hit multiplicity on the proton side, $N_{B B C p}$, versus forward calorimeter tower multiplicity, $N_{F C A L p}$ : the peak in the $(0,0)$ bin contains the DPE signal; (b) multiplicity distribution along the diagonal bins in (a) with $N_{B B C p}=N_{F C A L p}$; (c) $\xi_{\bar{p}}$ measured by the RPS for SD events (histogram) and for the "DPE" events of the $(0,0)$ bin in (a); (d) $\xi_{p}$ of the DPE events. In (c) and (d) the data are corrected for RPS acceptance on an event-by-event basis.

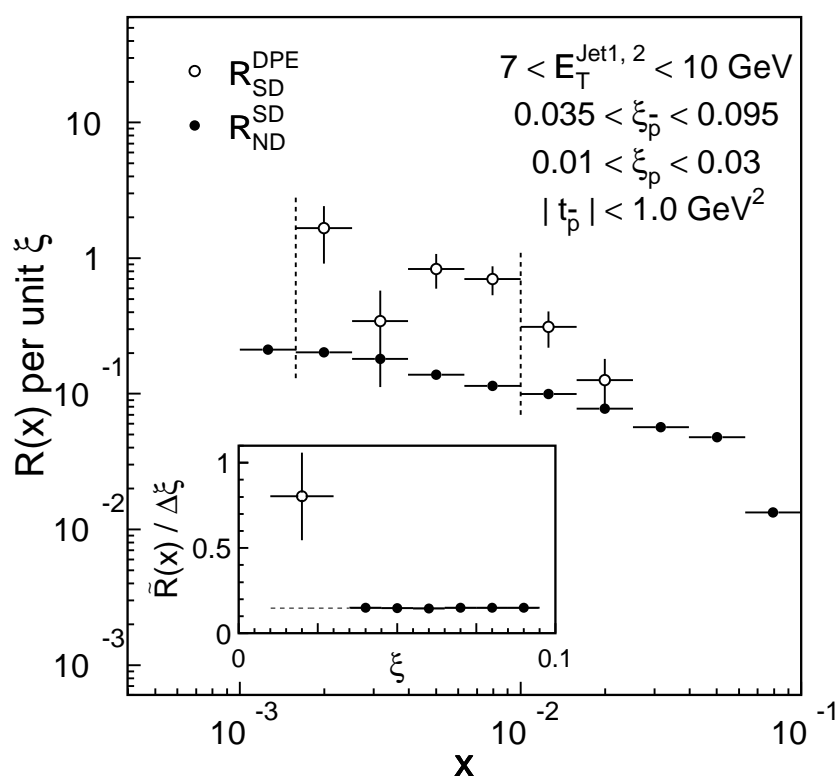

Figure 17. Ratios of DPE to SD (SD to ND) dijet event rates per unit $\xi_{p}\left(\xi_{\bar{p}}\right)$, shown as open (filled) circles, as a function $x$-Bjorken of partons in the $p(\bar{p})$. The errors are statistical only. The SD/ND ratio has a normalization systematic uncertainty of $\pm 20 \%$. The insert shows $\tilde{R}(x)$ per unit $\xi$ versus $\xi$, where the tilde over the $R$ indicates the weighted average of the $R(x)$ points in the region of $x$ within the vertical dashed lines, which mark the DPE kinematic boundary (left) and the value of $x=\xi_{p}^{\min }$ (right).

region of $x$ within the vertical dashed lines in the main figure. The ratio $\tilde{R}_{N D}^{S D}$, shown in six $\xi$ bins in the region $0.035<\xi<0.095$, is flat in $\xi$. A straight line fit to the six $\tilde{R}_{N D}^{S D}$ ratios extrapolated to $\xi=0.02$ yields $\tilde{R}_{N D}^{S D}=0.15 \pm 0.02$. The ratio of $\tilde{R}_{N D}^{S D}$ to $\tilde{R}_{S D}^{D P E}$ is $D \equiv \tilde{R}_{N D}^{S D} / \tilde{R}_{S D}^{D P E}=0.19 \pm 0.07$. The deviation of $D$ from unity represents a breakdown of factorization.

In Fig. 15, the presence of the rapidity gap on the antiproton side reduces the rapidity range over which a gap can be formed on the proton side. Thus, it appears that $D$ decreases as the $\eta$-range available for the formation of a rapidity gap increases. This behaviour was quantitatively predicted by the (re)normalized gap probability model 13. 


\section{CONCLUSIONS}

The central issue in hard diffractive production is the question of the existence of a unique, process independent diffractive structure function (DSF). This question has been addressed by CDF in three types of studies:

(a) Using forward rapidity gap (RG) tagging, the SD to ND ratios, $R_{N D}^{S D}$, for $W$, dijet, $b$ and $J / \psi$ production were found to be approximately the same in all cases, indicating that the partonic composition of the Pomeron (quark to gluon fraction) is similar to that of the proton. The measured values of $R_{N D}^{S D}$ at $\sqrt{s}=1800 \mathrm{GeV}$ are $\sim 10$ times smaller compared to predictions based on HERA measurements, indicating a breakdown of factorization.

(b) Using leading antiproton (LA) tagging, the DSF was measured from dijet production as a function of $\xi$ and $x_{b j}$. The results disagree both in shape and normalization with predictions based on extrapolations from HERA measurements, confirming the breakdown of factorization found in the RG studies and extending it to the shape of the DSF.

(c) Using a combination of LA tagging the same data sample as in (b)] and RG tagging on the proton side, a DPE signal was observed and the DSF was measured on the proton side. Comparing the proton DSF in DPE with the $\bar{p}$ DSF measured in SD, a breakdown of factorization was observed, similar in magnitude to that observed in SD between Tevatron and HERA.

In pursuing the underlying physics reason for the factorization breakdown in diffractive processes, it is interesting to note that the suppression in the normalization of the DSF increases with the rapidity phase space available for $R G$ formation, as foreseen in the RG renormalization model [13].

\section{REFERENCES}

1. This article contains excerpts from a paper presented by this author at the "International Symposium of Multiparticle Dynamics, Frascati, Italy, 8-12 September 1997" [Nuc. Phys. B (Proc. Supp) 71 (1999) 368-377] and from
Refs. [6.7].

2. See P.D.B. Collins, An Introduction to Regge Theory and High Energy Physics (Cambridge University Press, Cambridge 1977).

3. F. Abe et al., CDF Collaboration, Observation of diffractive $W$-boson production at the Fermilab Tevatron, Phys. Rev. Lett. 78 (1997) 2698.

4. F. Abe et al., CDF Collaboration, Measurement of diffractive dijet production at the Fermilab Tevatron, Phys. Rev. Lett. 79 (1997) 2636.

5. T. Affolder et al., CDF Collaboration, $O b$ servation of diffractive b-quark production at the Fermilab Tevatron, Phys. Rev. Lett. 84 (2000) 232.

6. T. Affolder et al., CDF Collaboration, Diffractive dijets with a leading antiproton in $\bar{p} p$ collisions at $\sqrt{s}=1800$ GeV, Phys. Rev. Lett. 84 (2000) 5043.

7. T. Affolder et al., CDF Collaboration, Dijet production by double Pomeron exchange at the Fermilab Tevatron, Phys. Rev. Lett. (accepted for publication).

8. H1 Collaboration, T. Ahmed et al., Phys. Lett. B 348 (1995) 681; C. Adloff et al., Z. Phys. C 76 (1997) 613.

9. ZEUS Collaboration, M. Derrick et al., Z. Phys. C 68, (1995) 569; Phys. Lett. B 356 (1995) 129; Eur. Phys. J. C 6 (1999) 43.

10. F. Abe et al., CDF Collaboration, The Collider Detector at Fermilab, Nucl. Instrum. Meth. A 271 (1988) 387; Amidei et al., ib. A 350 (1994) 73.

11. P. Bruni and G. Ingelman, in Proceedings of the International Europhysics Conference on High Energy Physics, Marseille, France, 2228 July 1993, edited by J. Carr and M. Perrottet (Editions Frontières, Gif-sur-Yvette, France, 1994) p.595.

12. F. Abe et al., Phys. Rev. D 50 (1994) 5518; D 50 (1994) 5535; D 50 (1994) 5550.

13. K. Goulianos, Renormalization of hadronic diffraction and the structure of the pomeron. Phys. Lett. B 358 (1995) 379. 